\title{
Ocorrência de Polypedilum (Tripodura) amataura Bidawid- Kafka, 1996 (Diptera; Chironomidae) em Aquascypha bydrophora (Berk.) Reid (Fungi; Stereaceae), com descrição da pupa na Amazônia Central, Brasil
}

Arlindo SERPA-FILHOํㅜㄹ Ruth Leila Menezes FERREIRA², Ulysses Carvalho BARBOSA².

\section{RESUMO}

Quatro larvas foram coletadas em água retida pelo fungo Aquascypha hydrophora (Berk.) Reid (Fungi: Stereaceae) fixo em troncos de árvores eretas, na Reserva Florestal Adolpho Ducke, Amazonas, Brasil. Das larvas coletadas, apenas um exemplar desenvolveu até a fase adulta, sendo identificado como Polypedilum (Tripodura) amataura Bidawid-Kafka, 1996. Esta é a primeira descrição para uma forma imatura deste gênero, e também o primeiro registro em micotelmata.

\section{PALAVRAS-CHAVE}

Polypedilum, Chironominae, Aquascypha, Amazônia Central.

\section{Occurrence of Polypedilum (Tripodura) amataura Bidawid-Kafka, 1996 (diptera; chironomidae) in Aquascypha hydrophora (Berk.) Reid (Fungi; Stereaceae), with description of the pupae in the Central Amazonian, Brazil}

\section{ABSTRACT}

Four larvae were collected in water with the fungus Aquascypha hydrophora (Berk.) Reid (Fungi: Stereaceae), fixed in tree trunks found in the Adolpho Ducke Forest Reserve, Amazonas, Brazil. Only one specimem reached the adult phase, which was identified as Polypedilum (Tripodura) amataura Bidawid-Kafka, 1996. This is the first description of an immature form of this genus, and also the first registered in mycotelmata.

\section{KEY-WORDS}

Polypedilum, Chironominae, Aquascypha, Amazonian Central.

${ }^{1}$ Laboratório de Referência Nacional em Simulídeos e Oncocercose do Instituto Oswaldo Cruz-IOC. Av. Brasil, 4365-Manguinhos-RJ. Pav.108,sala 9, CEP.21045-900. serpa@ioc.fiocruz.br

2 Coordenação de Pesquisas em Entomologia-(INPA).ruth@inpa.gov.br; ulysses@inpa.gov.br 


\section{INTRODUÇÃO}

Chironomidae Macquart, 1838 é a maior e mais freqüente família de insetos de águas continentais (Pinder, 1986), com elevada diversidade e ampla distribuição zoogeográfica (Reiss, 1981; Ashe, 1983; Ashe et al., 1987; Armitage et al., 1995). Os imaturos podem ser encontrados em diversos ambientes aquáticos tais como águas empoçadas, reservatórios, rios, riachos, lagos de várzea, lagoas, igarapés, igapós, além de micro-habitats que acumulam água de chuvas, tais como fitotelmatas e micotelmatas (Frank \& Lounibos, 1983).

O gênero Polypedilum Kieffer, 1912, apresenta uma distribuição bem ampla em todo o mundo, com exceção da Antártica. E segundo Maschwitz \& Cook (2000), possui cerca de 100 espécies em toda a Região Holártica. De acordo com Pinder \& Reiss (1983) larvas de Polypedilum Kieffer, 1912 ocupam grande variedade de ambientes aquáticos, utilizando diferentes substratos e alguns, plantas com água.

Os estágios imaturos do gênero podem ser encontrados em uma grande variedade de ambientes aquáticos (Maschwitz \& Cook, 2000), e as pupas são identificadas através da coloração sempre constante, com algumas variações de coloração para as espécies. As estruturas do corpo da exúvia da larva apresentam dificuldades na observação morfológica, diferentemente da análise taxonômica da exúvia pupal, onde a sua morfologia é bem evidente.

Os adultos do gênero são facilmente reconhecidos pela forma triangular do oitavo segmento abdominal, pelo pulvilo bífido e pelo gosnostilo robusto.

$\mathrm{Na}$ Amazônia, Ferreira et al. (2001) assinalam em Aquascypha hydrophora (Berk.) Reid, 1965, adultos de Coleoptera - Dysticidae e imaturos de três famílias de Diptera - Tipulidae, Chironomidae e Culicidae, esta última com nove espécies, o que mostra a aptidão desta micotelmata como criadouro para diversificada entomofauna.

A presença de Polypedilum e de outros Chironomidae em fitotelmatas é apresentada em vários trabalhos. Em um tipo de fitotelmata, Musa sp. na Costa Rica, Lichtwardt (1994) assinalou a presença de larvas de Chironomidae do gênero Polypedilum Kieffer, 1912.

$\mathrm{Na}$ Amazônia Central, somente duas espécies de Polypedilum foram descritas, a partir de machos adultos, P. (Polypedilum) ge Bidawid \& Fittkau, 1995 e P. (Tripodura) amataura BidawidKafka, 1996, ambos coletados no igarapé Barro Branco na Reserva Florestal Adolpho Ducke, Manaus, Amazonas. No entanto, até o momento seus imaturos são desconhecidos.

Este trabalho tem como objetivo de ampliar o conhecimento do gênero Polypedilum na Amazônia Central, para isso é descrita a pupa de $P$. (Tripodura) amataura.

\section{MATERIAL E MÉTODOS}

As coletas foram realizadas na Reserva Florestal Adolpho Ducke, que pertence ao Instituto Nacional de Pesquisas da Amazônia - INPA, Manaus, Amazonas (0255’S 5959’ W).

Os fungos Aquascypha hydrophora foram localizados em uma área adjacente ao igarapé do Acará, fixados em troncos de árvores mortas (Figuras $4 \mathrm{e} 5$ ). Os fungos apresentam estruturalmente a forma de um cálice, com diâmetro de $9,2 \mathrm{~cm}(2,7-14,9 \mathrm{~cm})$ e altura média de $16,3 \mathrm{~cm}(6,1-18 \mathrm{~cm})$, podendo comportar em média $35 \mathrm{ml}$ de água (1-70 ml). Segundo Reid (1965), estas micotelmatas são encontradas na região Norte do Brasil, Colômbia, Suriname, Guiana Francesa, Panamá e Venezuela.

O material retido nos receptáculos foi retirado com o auxílio de pipetas e acondicionado em recipientes plásticos de $120 \mathrm{ml}$ para transporte. A água do criadouro apresentou temperatura de $27^{\circ} \mathrm{C} \mathrm{e} \mathrm{pH} \mathrm{4,4.} \mathrm{Os} \mathrm{imaturos} \mathrm{de} \mathrm{Chironomidae} \mathrm{coletados} \mathrm{foram}$ acondicionados em recipientes de plástico com água do local onde foram coletados e levados para o laboratório visando emergência do imago, para facilitar sua identificação taxonômica. O material coletado, não foi corretamente individualizado pela equipe e somente um exemplar que sobreviveu chegou à imago, após dois dias. No trajeto do campo, foi notado que duas larvas sofreram predação por parte dos demais organismos aquáticos presentes no mesmo biótopo antes de serem triadas. Das larvas que sobreviveram, duas que foram individualizadas, uma não prosseguiu o desenvolvimento em decorrência da presença de fungos na água utilizada para a criação. Tais problemas inviabilizaram um número maior de exemplares para o estudo, como por exemplo, a descrição do estágio larval. Da única larva que permaneceu viva e chegou ao estágio adulto, obtivemos apenas a exúvia pupal e o adulto emergido que serviram de base para a identificação do gênero Polypedilum.

A exúvia pupal e a genitália do macho emergido foram montadas em Euparal, de acordo com Schlee (1966). Os materiais entomológico e botânico estão depositados na Coleção de Invertebrados e no Herbário do Instituto Nacional de Pesquisas da Amazônia - INPA, respectivamente.

\section{RESULTADOS}

O macho emergido foi identificado como Polypedilum (Tripodura) amataura, seguindo a descrição de sua pupa:

Polypedilum (T.) amataura BIDAWID-KAFKA, 1996.

\section{(FIGS.1-3)}

Pupa: 4,5 mm de comprimento e 1,5 mm de largura; coloração da exúvia castanho-clara; conjuntivo seguindo o padrão de coloração dos segmentos abdominais (Fig. 1). Cefalotórax: Corno torácico bem desenvolvido, com o formato afilado e achatado e com cerca de 12 ramificações; anel basal elíptico, 
posicionado para região anterolateral; membrana externa sem espinhos (cerdas torácicas não observadas claramente); lóbulo basal com formato semicircular; tubérculo pré-alar ausente. Abdome: (Fig. 1) Conjuntivos entre os S I-II, apresentando chagrin; entre os segmentos III IV-V, chagrins não muito visíveis e com uma ou duas fileiras de cerdas bem pontiagudas; tergito I com cicatriz bem visível; chagrin presente dos S III ao VIII, com seqüência de espinhos pontiagudos dispostos entre 2-4 fileiras dos S II ao IV e formando desenhos disformes; lateralmente no S VIII presença de uma área com coloração castanho-escura; em cada lado de S VII e VIII, foi observada a presença de quatro cerdas; S VIII com esporão postero-lateral robusto, com cerca de 0,052 mm (Fig. 3), apresentando diversos sub-dentes pontiagudos laterais; Cerdas abdominais (não observadas corretamente em cada um dos segmentos). Lóbulo anal: (Fig. 2) castanho-claro seguindo o padrão dos segmentos abdominais; com 0,10 mm de comprimento e 0,12 $\mathrm{mm}$ de largura; formato semi-circular, não possuindo chagrin na área interna e externa; 64 cerdas longas e dispostas em torno do lobo anal, cada uma medindo 0,8 mm; presença de duas cerdas teniadas com 0,19 $\mathrm{mm}$ na parte inferior do lobo, cada cerda maior que o comprimento do lobo anal.

Material examinado: BRASIL, Amazonas: Manaus, (Reserva Florestal Adolpho Ducke, Igarapé do Acará), exúvia pupal e B\&, IX.1998, R.L.M. Ferreira, J. Silva \& E. S. Pereira cols. (INPA).
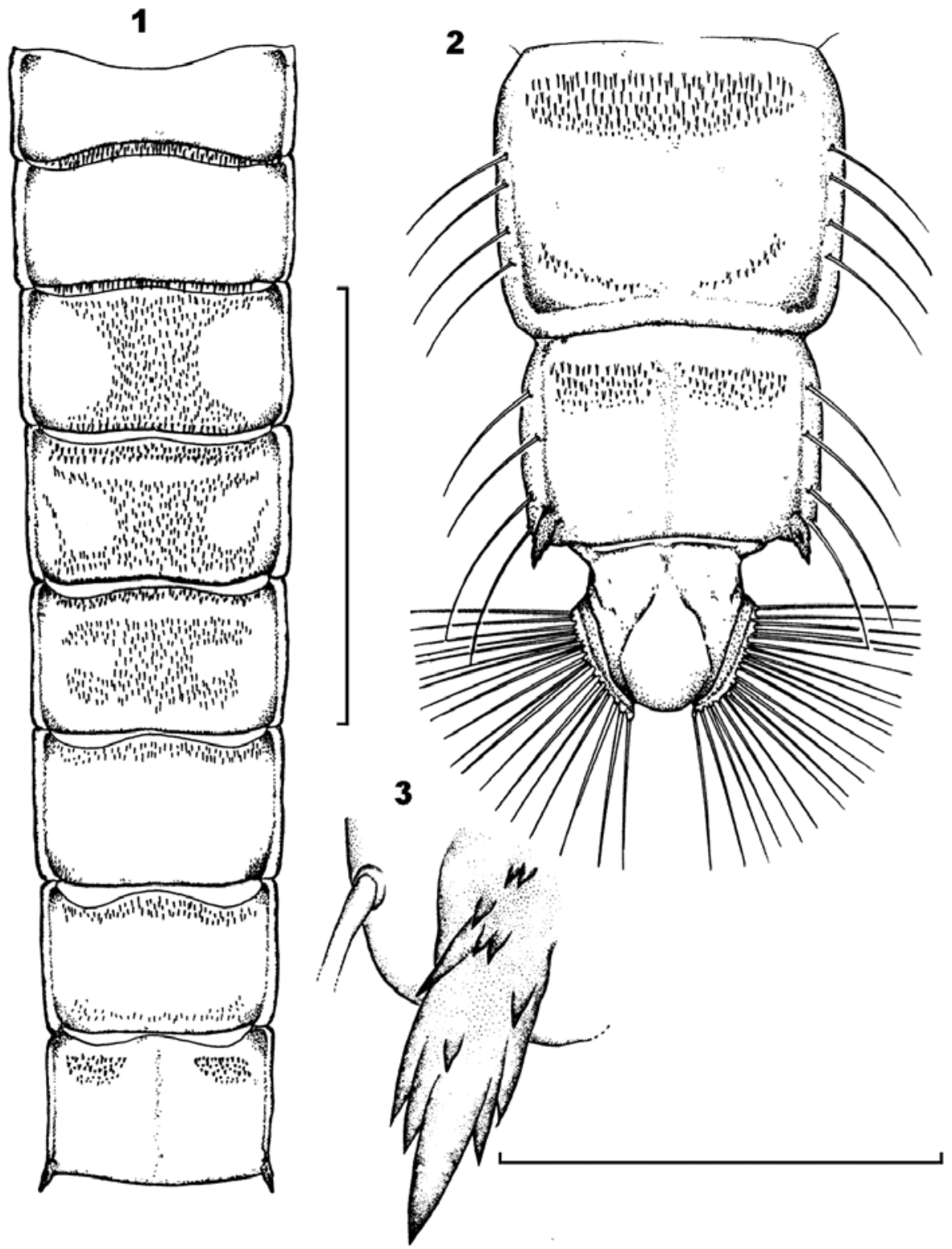

Figuras 1-3 - Polypedilum (T.) amataura Bidawid \& Kafka, 1996: Pupa. 1. Vista dorsal do abdome $(0,1 \mathrm{~mm})$; 2. últimos segmentos abdominais; $(0,1$ $\mathrm{mm})$; 3. esporão no penúltimo segmento anal. Escalas: 0,05 mm. 

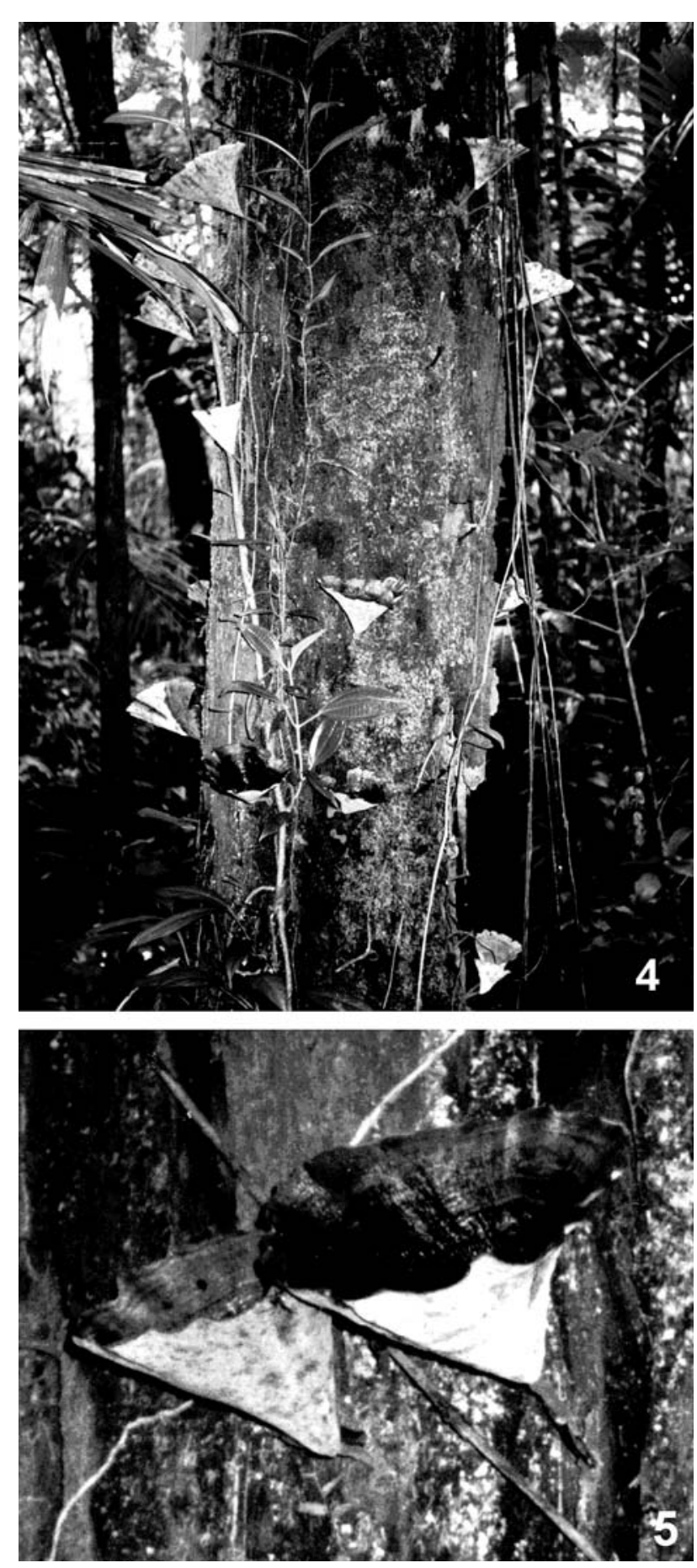

Figuras 4-5 - 4. Aquascypha hydrophora (Berk.) Reid (Fungus: Stereaceae) em tronco ereto; 5 . Detalhe do fungo com acúmulo de água.

\section{DISCUSSÃO}

Espécies de Chironomidae já foram registradas em fitotelmatas, desde os trabalhos de Picado (1913) na Costa Rica, e de Laessle (1961) na Jamaica, onde destacaram a presença destes dípteros em axilas das bromélias Tillandsia utriculata e Aechmea lingulata. Jones (1916) destaca a presença de um Metriocnemus edwardsi em plantas carnívora, Darlingtonia californica. Miller (1971) assinala a presença de larva de Chironomidae - Metriocnemus edwardsi em axilas de bromeliáceas. Kitching (1972) apontou a presença do mesmo gênero habitando buracos de arvores. Entretanto, foi com os trabalhos mais completos de Loden (1974) e Fish (1976), que os estudos de Chironomidae em fitotelmatas tomaram um impulso maior, sendo assinalada a presença de imaturos de Ablabesmyia, Monopelopia, Pentaneura pertencentes à subfamília Tanypodinae; Cryptochironomus, Tanytarsus, Metriocnemus, Chironomus da subfamília Chironominae e ainda algumas espécies da subfamília Orthocladiinae.

Cranston \& Judd (1987) contribuíram com a descrição de uma espécie nova de Metriocnemus yaquina, habitando fitotelmatas.

Richardson et al. (2000) comparam as faunas de fitotelmatas e citam a presença de larvas Chironomidae e Orthocladiinae sp. em Heliconia inflorescence e de Eukieferiella sp., exemplares de Tanytarsini sp. e de outros Chironomidae sem uma identificação mais precisa, em Bromélias. Os mesmos autores mencionam ainda que encontraram pupas de chironomidae próximas ao gênero Stempellinella, além de outras pupas de Chironominae que não foram identificadas.

Em Kitching (2001), foi assinalada a presença de Chironomidae em cinco tipos de fitotelmatas, tendo sido apontada presença das subfamílias Podonominae, Chironominae, Orthocladiinae, Tanypodinae, com identificação de apenas um gênero: Metriocnemus, sendo observada também entre estes a capacidade predatória e também decompositora.

Apesar da gama de trabalhos existentes sobre Chironomidae em fitotelmatas, somente no trabalho de Fish (1976), foi dada uma importância maior para os estudos com micotelmatas. Até o momento quase nada se conhece sobre a taxonomia e a ecologia de exemplares de Chironomidae encontrados neste tipo de microambiente. No trabalho de Ferreira et al. (2001), foi assinalada a presença de imaturos de Chironomidae em micotelmatas, mas o mesmo não foi descrito, sendo, portanto, esta a primeira descrição sobre um Chironomidae encontrado em Micotelmata no mundo.

Este tipo de Micotelmata se caracteriza por apresentar, segundo Reid (1965), "os esporóforos com 4-12 cm de altura, de 3,5-15 cm de diâmetro; pileo coriáceo centralmente, forma afunilada; pileus finamente tomentoso ou aveludado, com variação na coloração, indo de marrom escuro a violeta escura. Superficie do himênio geralmente zoneada concentricamente, com pruinosidade distinta; estipe apresenta 1,5-3 cm de comprimento e 0,3-0,5 de largura, relativamente curto".

Atualmente, existe registro de 64 espécies para o gênero Polypedilum na região neotrópica (Spies \& Reiss, 1996), com 
descrições apenas dos adultos sem oferecer informaçōes detalhadas sobre os imaturos destas espécies.

Estudos com imaturos do gênero Polypedilum na região Amazônica ainda são insuficientes. Não são encontrados trabalhos completos sobre imaturos de Polypedilum e os mesmos se restringem aos de Bidawid \& Fittkau (1995) e Bidawid - Kafka (1996), com descrição de 30 espécies novas do subgênero $P$. (Polypedilum) e 34 de $P$. (Tripodura). No Brasil, pouco se sabe sobre os Chironomidae de Fitotelmatas e principalmente das Micotelmatas.

Recentemente, os trabalhos de Mendes et al. (2003), Pinho et al. (2005), Marcondes \& Pinho (2005), com a fauna chironomidológica de fitotelmatas, vêem valorizando bastante a importância da associação de imaturos com os adultos encontrados nestas plantas. Porém, em diversos trabalhos sobre fitotelmatas ainda são feito apenas registros sobre a presença de Chironomidae, com a indicação dos estágios de desenvolvimento, não havendo uma preocupação maior para a identificação ao menor nível taxonômico, o que impede um maior conhecimento sobre a ecologia, biologia e principalmente a taxonomia dos exemplares que utilizam estes vegetais para o seu desenvolvimento.

A identificação do exemplar adulto deste trabalho foi corroborada devido a comparação com os trabalhos de Bidawid \& Fittkau (1995) e Bidawid - Kafka (1996). A identificação foi facilitada pela presença de algumas características morfológicas, como: (1) a presença de cerdas bem definidas no gonostilo; (2) a forma da ponta anal - fina e comprida; (3) a volsela, que seguiu o mesmo padrão marcante descrito para a ponta anal; assim como (4) as asas totalmente hialinas e com as nervuras que definem muito bem o gênero estudado.

Já para a pupa, de uma forma geral, Polypedilum são identificados pela presença de corno torácico com muitas brânquias, abdome sem pigmentos, mas com chagrin bastante forte e esporão bem proeminente posicionado na porção lateral do tergito (Wiederholm, 1986; Bjorlo et al., 2000; Maschwitz \& Cook, 2000). A exúvia pupal de Polypedilum (T.) amataura, descrita neste trabalho, apresenta o esporão no VIII tergito, proeminente, o desenho do chagrin bem definido e presença de numerosas cerdas do lobo anal.

Ë importante ressaltar que até o momento, sabemos que imaturos de Polypedilum são facilmente encontrados em diversos substratos ou sedimentos e freqüentemente associados à vegetação aquática apresentando grande capacidade de adaptação (Maschwitz \& Cook, 2000). Ao assinalarmos esta espécie vivendo neste tipo de ambiente particular apresentamos Aquascypha hydrophora (Fungi; Stereaceae), como uma outra forma de ambiente onde ocorre o desenvolvimento de Polypedilum.

\section{AGRADECIMENTOS}

Ao MSc. Stefan Keppler pela tradução da bibliografia em alemão e a Dayse Cristina e André Araújo, pela arte final das estampas.

\section{BIBLIOGRAFIA CITADA}

Armitage, P.D.; Cranston, P.S.; Pinder, L.C.V. 1995. The Chironomidae Biology and ecology of non-biting midges, 1st ed., Chapman \& Hall, London, 538pp.

Ashe, P. 1983. A catalogue of chironomid genera and subgenera of the world including synonyms (Diptera: Chironomidae). Entomologica Scandinavica, 20(Suppl.): 1-68.

Ashe, P; Murray D. A.; Reiss, F. 1987. The zoogeographical distribution of Chironomidae (Insecta: Diptera). Annales Limnologie, 23 (1): 27-60.

Bidawid, N.; Fittkau, E.J. 1995. Zur Kenntnis der neotropischen Arten der Gattung Polypedilum Kieffer, 1912. Teil I. (Diptera, Chironomidae). Entomofauna, 16(11): 465-536.

Bidawid-Kafka, N. 1996. Zur Kenntnis der neotropischen Arten der Gattung Polypedilum Kieffer, 1912. Teil II. (Diptera, Chironomidae). Entomofauna, Ansfelden, 17(11): 165-240.

Bjørlo, A.; Vårdal, H.; Saether, O. A. 2000. A preliminary phylogenetic analysis of the subgenus Tripodura Twones of the genus Polypedilum Kieffer, 1912 (Diptera, Chironomidae). In: Hoffrichter, O. (Ed.) Late 20th Century Research on Chironomidae: an Anthology from the 13th International Symposium on Chironomidae, p.103-127.

Cranston, P.S.; Judd, D.D. 1987. Metriocnemus (Diptera: Chironomidae) - an ecological survey and description of a new species. Journal of the New York Entomological Society 95: 534546.

Ferreira, R.L.M.; Oliveira, A.F.; Pereira, E. S.; Hamada, N. 2001. Occurence of Larval Culicidae (Diptera) in Water Retained in Aquascypha hydrophora (Fungus: Stereaceae) in Central Amazonia, Brazil. Memórias do Instituto Oswaldo Cruz, 96(8): 1165-1167.

Fish, D.N. 1976. Structure and composition of the aquatic invertebrates community inhabiting epiphytic bromeliads in south Florida and the discovery of an insectivorous bromeliad. PhD's Thesis, Univ. Florida. 78pp.

Frank, J.H.; Lounibos, L.P. 1983. Phytotelmata: Terrestrial Plants as Host for Aquatic Insect Communities. Plexus, Medford, New Jersey. 293pp.

Jones, F. M. 1916. Two insect associates of the California pitcher plant Darlingtonia californica. Entomological News, 27: 385-392.

Kitching, R.L. 1972. Population studies of the immature stages of the tree-hole midge Metriocnemus martini Thienemann (Diptera: Chironomidae). Journal of Animal Ecology, 41: 53-62.

Kitching, R.L. 2000. Food webs and container habitats: the natural history and ecology of phytotelmata. Cambridge; Cambridge Univ. Press, $431 \mathrm{pp}$.

Kitching, R.L. 2001. Food webs in phytotelmata: "bottom-up" and "top-down" explanations for community structure. Annual Review of Entomology, 46: 729-760.

Laessle, A.M., 1961. A micro-limnological study of Jamaican Bromeliads. Ecology, 42: 499-517. 
Langton, P.H. 1995. The pupa and events leading to eclosion. In: Armitage, P.D.; Cranston, P.S.; Pinder, L.C.V. (eds.): The Chironomidae. Biology and ecology of non-biting midges, Chapman Hall, p. 169-193.

Lichtwardt, R.W.1994. Trichomycete fungi living in the guts of Costa Rican phytotelm larvae and other lentic dipterans. Revista de Biología Tropical, 42(1/2): 31-48.

Loden, M.S. 1974. Predation by Chironomid larvae on Oligochaetes. Limnology and Oceanography, 19:156-159.

Marcondes, C.B.; Pinho, L.C. 2005. First description of an emergence trap for bromeliads and preliminary results of collections from southern Brazil (Insecta: Diptera and others). Studia Dipterologica, 12(1): 3-7.

Maschwitz, D.E.; Cook, E.F. 2000. Revision of the Nearctic Species of the Genus Polypedilum Kieffer (Diptera: Chironomidae) in the Subgenera P. (Polypedilum) Kieffer and P. (Urespedilum) Oyewo and Saether. Ohio Biological Survey Bulletin (New Series) 12 (3): 135pp.

Mendes, H.F.; Marcondes, C.B.; Pinho, L.C. 2003. A new phytotelmatic species of Monopelopia Fittkau, 1962 (Insecta: Diptera: Chironomidae: Tanypodinae) from South Brazil. Zootaxa, 262: 1-10.

Miller, A.C. 1971. Observations on the Chironomidae (Diptera) inhabiting the leaf axils of two species of Bromeliaceae on St. John, U.S. Virgin Islands. Canadian Entomologist 103: 391-396.

Picado, C. 1913. Les broméliacées épiphytes considérées comme milieu biologique. Bulletin Scientifique France et Belgique 5:215360.

Pinder, L.C.V. 1986. Biology of freshwater Chironomidae. Annual Review of Entomology, Stanford, 31: 1-23.
Pinder, L.C.V.; Reiss, F. 1983. The Larvae of Chironomidae (Diptera: Chironomidae) of the Holoarctic region - Keys and diagnoses. In: Wiederholm, T. (Ed.). Chironomidae of the Holoarctic region. Part. 1. Larvae. Entomologica Scandinavica, Supplement, 19: 293-435.

Pinho, L.C.; Mendes, H.F.; Marcondes, C. B. 2005. A new Brazilian species of Stenochironomus Kieffer mining deacying leaves in bromeliads (Diptera: Chironomidae). Zootaxa, 1036: 37-47.

Reid, D.A. 1965. A monograph of the Stipitate Stereoid Fungi. Weinhein. Vertag Von J. Cramer. 367pp.

Reiss, F. 1981. Chironomidae, p. 261-268. In: Hurlbert, S.H.; Rodriguez, G.; Santos, N.D. (eds.). Aquatic biota of tropical South America. Part 1. Arthropoda, San Diego State University, San Diego, California. 333pp.

Richardson, B.A., Rogers, C., Richardson, M.J. 2000. Nutrients, diversity, and community structure of two phytotelm systems in a lower montane forest, Puerto Rico. Ecological Entomology, 25: 348-356.

Schlee, D. 1966. Präparation und ermittlung von messwerten an chironomidae (Diptera). Gewäss Abwäss, 41/42: 169-193.

Spies, M.; Reiss, F. 1996. Catalog and bibliography of Neotropical and Mexican Chironomidae (Insecta, Diptera). Spixiana, 22 (suppl): 61-119.

Varga, L. 1928. Ein interesanter Biotop der Biocönose von Wasserorganismen. Biological Zentralblatt, 48: 43-162.

Wiederholm, T. (ed.) 1986. Chironomidae of the Holarctic region. Keys and Diagnoses. Part II. Pupae. Entomologica Scandinavica Supplement, 28: 1-482.

Recebido em 29/07/2005

Aceito em 12/02/2007 